\title{
Management of varicella zoster virus exposure and infection (chickenpox) in pregnancy and neonates
}

\author{
Rohini Fernandopulle ${ }^{1}$, Shalini Sri Ranganathan ${ }^{2}$ \\ Sri Lanka Journal of Child Health, 2001; 30: 111-5
}

(Key words: Varicella-zoster virus infections in pregnancy, adverse effects)

\section{Introduction}

Varicella-zoster virus infections in pregnancy can cause severe adverse effects in the mother, fetus and neonate, which vary with the period of gestation. For the mother the adverse effects are greatest in the second half of pregnancy whereas for the fetus it is greatest in the first and second trimester.

\section{Risk to the pregnant mother}

If the pregnant mother has had chickenpox previously there is no risk to the mother or baby. If mother gets chickenpox in pregnancy there is some evidence, though scant, that it is more severe than in non-pregnant adult, and of increased severity in the second half of pregnancy ${ }^{1}$. Pneumonia is the most common complication in adults and can be severe in pregnancy ${ }^{1,2,3}$. Therefore, evaluation of the mother's immune status is important and involves careful history taking and serologic evaluation. Although, we do not have local data, most mothers (up to 95\%) with uncertain histories are immune especially if other family members had chickenpox and the mother was in the family at that time. It is also important to ask whether she was exposed to other people with chickenpox when she was younger and what happened. If uncertain do a serologic evaluation if the facility is available. There is no evidence that terminating the pregnancy speeds maternal recovery from chickenpox.

Risk to the fetus if the mother gets chickenpox before the 20th week of pregnancy ${ }^{1,2,3}$

It does not increase the risk of spontaneous abortion.

There is no indication for induced abortion.

Fetal infection is usually benign and self-limiting. Occasionally it can cause a characteristic syndrome "congenital varicella syndrome" (CVS) first described in 1947. The typical features include hypoplasia of one limb, cicatricial lesions with a dermatomal distribution

${ }^{1}$ Senior Lecturer in Pharmacology, ${ }^{2}$ Lecturer in Pharmacology, Faculty of Medicine, Colombo. affecting the same limb, neurological abnormalities (hydrocephalus and microcephaly), eye abnormalities (Horner's syndrome, cataract, choroidoretinitis, microphthalmia), functional or structural defects of the gastrointestinal or genitourinary tracts, and growth retardation. The risk of embryopathy is about $0.4 \%$ until 14 weeks and $1-2 \%$ from $14-20$ weeks ${ }^{1,2,3}$.

At present there is no reliable marker of in-utero infection or to predict CVS development.

Risk to the fetus if the mother gets chickenpox after 20th week of pregnancy to 7 days before delivery ${ }^{1,2,3}$.

Varicella infection more than seven days before delivery ensures adequate transplacental passage of specific antibodies to protect the infant.

There is no risk of malformation

Risk to the neonate if mother gets chickenpox in the 7 days before delivery to 28 days after delivery ${ }^{1,2,3}$.

The timing of maternal chickenpox in relation to delivery determines the degree of risk to the newborn baby. Approximately $50 \%$ of fetuses will be infected. Postnatal infection is usually mild, but can be serious in about $10 \%$ of newborn babies. The neonate is at highest risk of disseminated disease:-

- If the mother develops chickenpox during the period 7 days before to 2 days after delivery. It has a relatively short incubation period (11 days), with an onset in 5 to 10 days of age and presents with a rash at birth or develops it within a few days. It is attributed to a large transplacental innoculum of virus in the absence of protective maternal antibody (it takes about a week.for antibodies to appear in maternal plasma).

- If born at or before 28 weeks gestation or born weighing under $1000 \mathrm{~g}$ regardless of the immune status of the mother.

Therefore, if possible delay delivery to allow time for antibodies to appear in the maternal plasma. 
A full term baby of a mother who develops chicken pox after the first 2 days and within the first 28 days after delivery is at a lower risk of disseminated disease but the risk is higher when compared with older infants.

Herpes zoster occurs in early childhood in about $1 \%$ of babies exposed to maternal chickenpox during the second half of pregnancy.

\section{Drugs}

The prophylactic use of zoster immunoglobulin (ZIG) and timely use of aciclovir is known to prevent or reduce the severity of chickenpox in both mother and neonate.

A recent study on varicella in pregnancy indicates a possible reduction in materno-fetal transmission of varicella infection following ZIG after contact ${ }^{2}$. Pregnant women are the only healthy adults for whom ZIG is made available ${ }^{1,2}$. ZIG can prevent or modify clinical varicella if given within 72 hours (latest 96 hours). There is no evidence that it prevents fetal infection or damage. Therefore close ultrasound monitoring and counselling is recommended. ZIG is not readily available, hence we recommend intravenous pooled normal immunoglobulin (IVIG) although it is not a licensed indication, followed by prophylactic aciclovir in high risk situations. Exposed neonates should be given ZIG or IVIG or intramuscular pooled immunoglobulin (IMIG ) ${ }^{4}$ after assessing the risk.

Aciclovir is not licensed for use in pregnancy but there have been no adverse effects among hundreds of pregnant users over several years of monitoring. It can be used for prophylaxis and treatment of chicken pox. It should be given intravenous for severe illness and complications (Box 1) at any stage of pregnancy and neonatal period. The role of prophylactic aciclovir in neonates is unproven. For management protocols see Boxes 2 and 3.

\section{Isolation}

- Neonates with CVS are not contagious and do not need isolation

- An infected mother does not need to be isolated from her own baby. Breast feeding of infected or exposed babies should be encouraged.

- Quarantine of cases should continue until all lesions have crusted

\section{Box $1^{1,2}$}

\section{Significant exposure in pregnancy is defined as}

- Living in the same household as a person with active chicken pox or zoster

- $\quad$ Face to face contact with a person with chickenpox or uncovered zoster for at least 5 minutes

Risk factors for serious disease in pregnancy are

- Chronic lung disease

- Cigarette smoking

- Systemic corticosteroid therapy

- Impaired immunity

- Second half of pregnancy

Evidence of serious disease include

- Respiratory

- Neurological symptoms

- Haemorrhagic rash

- Continued fever or appearance of new lesions after 6 days from onset especially if associated with a cough 


\section{Box 2}

\section{Management of a pregnant woman exposed to or have clinical varicella ${ }^{1,2}$}

\section{Post exposure prophylaxis}

- If no previous reliable history or serological assay is negative and mother has had significant exposure (Box 1) to chickenpox or has high risk factors for serious disease (Box 1), give ZIG / IVIG within 72 hours (latest 96 hours). If ZIG is not readily available give intravenous normal pooled immunoglobulin (IVIG). If ZIG /IVIG is not given within 72 hours or is not given at all give prophylactic oral aciclovir, starting on day 7 after exposure.

- If mother is exposed to chickenpox and is in the third trimester consider prophylactic oral aciclovir starting on day 7 of exposure.

- In others close observation followed by prompt initiation of aciclovir on suspicion of chickenpox is recommended. If aciclovir is not initiated within 24 hours of the rash, monitor (in hospital, if in the second half of pregnancy) and give IV aciclovir if disease is progressive or complications develop.

- Dose -

ZIG - 125unit/10kg bodyweight (max adult dose 625units) or $6 \mathrm{ml}$,

IVIG $-4 \mathrm{ml} / \mathrm{kg}, 200 \mathrm{mg} / \mathrm{kg}$

Aciclovir - Oral 800mg five times a day for 7 days

\section{Treatment of chicken pox}

- Mild infection - oral aciclovir : 800mg five times a day for 7 days or 48 hours after the cessation of new lesions whichever is longer.

- Severe chickenpox or complications (Box 1) - Intravenous aciclovir $10-20 \mathrm{mg} / \mathrm{kg}$ every 8 hours for a minimum of 7 days followed by oral.

- There is wide clinical experience with the use of aciclovir in pregnancy, hence it is considered safe for use.

- $\quad$ ZIG / IVIG is ineffective and should not be given to the mother if chickenpox has developed.

- A pregnant woman who has varicella can deliver vaginally. Expedited delivery should only be considered for fetal compromise or if the gravid uterus is considered to critically impair maternal ventilation.

- Varicella vaccination is contraindicated 
Box 3

\section{Management of a neonate ( 0 - 4 weeks) exposed to or have chickenpox ${ }^{1,3}$}

\section{Post exposure prophylaxis}

- Administer ZIG / IVIG as early as possible after delivery or exposure but must be within 72 hours to newborns at high risk. ie.

- Neonates exposed to maternal chickenpox within 7 days or less before delivery or starting within 48 hours after delivery.

- Neonates born at or before 28 weeks gestation or born weighing under $1000 \mathrm{~g}$ regardless of the immune status of the mother.

- Infants born after 28 weeks gestation and mother is non immune should only be given ZIG if they have had significant exposure (see definition).

- Neonates whose mother develops chickenpox 3 days or more after delivery and within 28 days are also at risk but lower. The need for ZIG / IVIG should be individually assessed. Give IMIG $\{0.6-1.2 \mathrm{ml} / \mathrm{kg}$ (4) $\}$ and observe closely and initiate prompt treatment with aciclovir if chicken pox is suspected.

- Close follow up is needed even if given ZIG/IVIG /IMIG because severe disease can occur despite passive immunisation.

- Maternal zoster is not an indication for ZIG.

- If siblings get chicken pox, the risk to the neonate is negligible if the mother has had chicken pox. If not assess and give immunoglobulins.

- Parents should be advised to seek prompt medical attention if the baby develops a rash.

- The role of prophylactic aciclovir in neonates is unproven.

\section{Treatment of chicken pox}

- Only cutaneous vesicles in a low risk full term neonate -give oral aciclovir $20 \mathrm{mg} / \mathrm{kg} / \mathrm{every} 6$ hours for 7 days or 48 hours after the cessation of new lesions whichever is longer.

- IV aciclovir (10 mg/kg, 8 hourly) for a minimum of 7 days followed by oral upto 48 hours after the cessation of new lesions should be given to

- High risk neonates who did not receive ZIG or administration of ZIG was delayed beyond 24 hours.

- Ill neonates with chickenpox (poor feeding, tachypoeic)

$\circ$ Immunocompromised neonates 


\section{Conclusion}

We have attempted to provide guidelines for the rational use of the available interventions in the treatment and prophylaxis of chicken pox in the pregnant mother and newborn after analysing the available evidence and the local scenario.

\section{References}

1. Heuchan A. Issaacs D on behalf of the Australasian subgroup in Paediatric Infectious Diseases of the Australasian Society for Infectious Diseases. The management of varicella-zoster exposure and infection in pregnancy and the newborn. Medical Journal of Australia 2001; 174: 280-292.
2. Report of Expert Committee. In Workshop Report on the Treatment of Herpes Simplex, Varicella and Zoster. The Norwegian Medicines Control Authority and Medical Products Agency, Sweden. 1998; 3: 9-31.

3. Enders G, Miller E, Cradock- Watson J, Bolley I, Ridehalgh M. Consequences of varicella and her pes zoster in pregnancy; prospective study of 1739 cases. Lancet 1994; 343: 1548-51.

4. Canadian Pharmaceutical Association. Compendium of Pharmaceuticals and Specialities 1994. 
